\title{
Distilling Consciousness: Isolated the Underlying Brain Activity?
}

\author{
Antonella Macerollo ${ }^{1,2}$ and Graziella Quattrocchi ${ }^{1}$ \\ ${ }^{1}$ Sobell Department of Motor Neuroscience and Movement Disorders, University College London, London WC1N 3BG, United Kingdom, and ${ }^{2}$ Department \\ of Basic Medical Sciences, Neuroscience and Sense Organs, Aldo Moro University of Bari, 70121 Bari, Italy \\ Review of Li et al.
}

How does our brain build its representation of the world surrounding us? What are the neural correlates and mechanisms at the base of conscious experience? This old, yet timely, question is also one of the most challenging to investigate. Indeed, identifying the neural correlates of consciousness (NCC) requires one to disentangle these processes from those that are strictly related but without an actual conscious content. The lack of such discrimination is one of the weaknesses of the experimental paradigm classically used to study NCC, the "minimal contrast" approach (Baars, 1989; Dehaene and Changeux, 2011), in which stimulus conditions are held similar while conscious perception varies. Indeed, brain activity registered under the conscious condition may also reflect processes preceding or following conscious perception, but not responsible of it - the so-called "prerequisites" (NCC-pr) and "consequences” (NCC-co) of NCC (Aru et al., 2012).

Li et al. (2014) addressed this issue in a recent study published in The Journal of Neuroscience. The authors used magnetoencephalography (MEG) to record brain activity while participants performed a behavioral task designed to give a trial-by- trial measure of subjective awareness (SUB, i.e., stimulus reported as seen vs unseen), objective performance (OBJ, i.e., participants answered correctly vs incorrectly), and confidence of SUB (CONF, i.e., participants' confidence of being correct rated on a 4-point scale). This behavioral paradigm, a combination of liminal stimulation, forced alternative choice, and trial-by-trial introspection (Lamy et al., 2009; Fleming et al., 2010; Hesselmann et al., 2011), is one of the main strengths of this study, because it gives a separate quantitative measure for each one of the studied neural processes. Considering that NCC contributes by definition only to SUB; that pr-NCC contributes both to SUB and OBJ; and that NCC-co affects SUB and CONF (Aru et al., 2012), a separate measure for each one of these processes permitted the authors to associate the brain activity registered by MEG to specific processes. Furthermore, the isolation of brain activity related to SUB permitted the authors to investigate the correlation between slow cortical potentials (SCPs) measured by MEG (Leistner et al., 2007) and SUB, a link hypothesized by $\mathrm{He}$ and Raichle (2009).

The authors found enhanced longlasting event related fields when trials were both seen and correct (i.e., SUB and $\mathrm{OBJ})$, but not when they were unseen and correct (i.e., OBJ), or unseen and incorrect (i.e., no SUB, no OBJ). The authors thus hypothesized that this activity was related to SUB, which was confirmed with a three-way ANOVA on MEG activity taking into account SUB, OBJ, and CONF.

The identification of brain activity specific to SUB is a central contribution to the current research on the neural signatures of NCC. In addition, correlating MEG with MRI data, the authors investigated the anatomical locus and the timing of activity related to SUB and OBJ. The activity related to SUB, but not to OBJ, was most pronounced between $500 \mathrm{~ms}$ and $1.5 \mathrm{~s}$ after the stimulus, widespread in the frontoparietal and temporal areas, and slow (mainly in the 0.05-1 Hz band). This is a signature of SCPs, consistent with the theory of He and Raichle (2009), supporting an involvement of SCPs in the emergence of conscious awareness. In contrast, the CONF-related activity was relatively transient.

To investigate the cortical excitability state, which is not indicated by MEG recording, two participants underwent EEG recording under the same experimental conditions. Interestingly, in the seen and correct condition (SUB and OBJ), compared with the other conditions, a transient negative potential at $\sim 300 \mathrm{~ms}$, followed by a slow positive potential, with the same time window (500 $\mathrm{ms}$ to $1.5 \mathrm{~s}$ ) and anatomical distribution as the MEG activity related to SUB, was noted. Despite the very small sample size, these results are in accordance with what was observed with MEG, and suggest that SUB is correlated with cortical inhibition rather than excitation. If this is true, SCPs could be 
interpreted as a potential mechanism to reduce the flow of irrelevant information, thus enhancing the stimulus percept at the conscious level. In terms of anatomical distribution, these results are consistent with the hypothesis of widespread brain activity producing the emergence of consciousness, in accordance with the global neuronal workspace (GNW) theory of consciousness proposed by Dehaene and Changeux (2011).

We believe this study has two main strengths. First, the behavioral paradigm used gives a measure of some of the main processes related to NCC. This represents a clear advance in research on conscious experience. Li et al. (2014) applied a new innovative behavioral protocol that yielded a clear separation between NCC and NCC-co and this is a further step in understanding the NCC.

Second, the use of a high-pass filter around $0.05 \mathrm{~Hz}$ in the MEG recordings permitted the authors to analyze the slow long-lasting activity underlying conscious perception, which is strictly related to SCPs. In line with the GNW, the authors found a consistent link between late activity and awareness, meaning a time window of 200-600 ms after stimulus. In contrast, previous studies indicated a time window around 200-300 ms as a "primary candidate for a neural correlate of awareness" and the interval around $400-600 \mathrm{~ms}$ as activity related to further processes, such as working memory maintenance and, thus, part of co-NCC (Aru et al., 2012). Li et al. (2014) opened a new discussion on the time duration of the tripartite distinction of NCCs and this highlights the potential overlap among these activities.

In addition, we have to emphasize that Li et al. (2014) results were confirmed by a retest reliability performed in two partic- ipants through three MEG sessions, giving strong power to the results and indicating a late slow positive activity cortical inhibition related to SUB and, thus, manifestation of SCPs as a potential mechanism to reduce the flow of negligible information enhancing the stimulus percept at the conscious level.

Nevertheless, some limitations hamper our enthusiasm for this study. First, pr-NCC can be linked to a variety of processes, such as attention, decision bias, stimulus expectation, adaptation, working memory, and potentially other mechanisms still under investigation (Aru et al., 2012), which can influence both SUB and OBJ, and which are not directly measured by the used behavioral task. Furthermore, because sensor locations varied across MEG sessions, it was not possible to confirm the anatomical location of the studied processes in the retest. Finally, the observations of Li et al. (2014) are difficult to reconcile with some previous studies regarding the meaning of gamma power. In fact, Li et al. (2014) found that increased power in the gamma frequency correlated with OBJ but not with SUB, in line with Aru et al. (2012) and, thus, arguing against the previous link with SUB found by Gaillard et al. (2009). Further study of SCPs and consciousness should be encouraged to clarify these inconsistencies.

In conclusion, the study by $\mathrm{Li}$ et al. (2014) implicates long-lasting MEG activity, especially SCPs, in consciousness perception. Hence, this specific range of the brain electrical activity is a valuable target for future MEG studies to clarify the specific spatial pattern of the cortical areas involved in this cognitive process. Notably, the current work excluded a confounding effect of working memory decay on the results, but did not investigate the possible links between conscious percep- tion and several other cognitive processes. In particular, in the future, it would be interesting to investigate the potential roles of different forms of memory in the SUB to establish whether different cognitive processes are mediated by an overlapping spatiotemporal pattern of neural network activity.

\section{References}

Aru J, Bachmann T, Singer W, Melloni L (2012) Distilling the neural correlates of consciousness. Neurosci Biobehav Rev 36:737-746. CrossRef Medline

Baars BJ (1989) A cognitive theory of consciousness. Cambridge: Cambridge UP.

Dehaene S, Changeux JP (2011) Experimental and theoretical approaches to conscious processing. Neuron 70:200-227. CrossRef Medline

Fleming SM, Weil RS, Nagy Z, Dolan RJ, Rees G (2010) Relating introspective accuracy to individual differences in brain structure. Science 329:1541-1543. CrossRef Medline

Gaillard R, Dehaene S, Adam C, Clémenceau S, Hasboun D, Baulac M, Cohen L, Naccache L (2009) Converging intracranial markers of conscious access. PLoS Biol 7:e61. CrossRef Medline

He BJ, Raichle ME (2009) The fMRI signal, slow cortical potential and consciousness. Trends Cogn Sci 13:302-309. CrossRef Medline

Hesselmann G, Hebart M, Malach R (2011) Differential BOLD activity associated with subjective and objective reports during "blindsight" in normal observers. J Neurosci 31:12936-12944. CrossRef Medline

Lamy D, Salti M, Bar-Haim Y (2009) Neural correlates of subjective awareness and unconscious processing: an ERP study. J Cogn Neurosci 21:1435-1446. CrossRef Medline

Leistner S, Sander T, Burghoff M, Curio G, Trahms L, Mackert BM (2007) Combined MEG and EEG methodology for non-invasive recording of infraslow activity in the human cortex. Clin Neurophysiol 118:2774-2780. CrossRef Medline

Li Q, Hill Z, He BJ (2014) Spatiotemporal dissociation of brain activity underlying subjective awareness, objective performance and confidence. J Neurosci 34:4382-4395. CrossRef Medline 